Prof. Dr. rer. nat. Jürgen Vormann, Ismaning, Deutschland

\section{Ernährungsbedingte Risiken sind die wichtigsten Faktoren für chronische Erkrankungen}

Die Bedeutung von chronischen Erkrankungen hat in den vergangenen Jahrzehnten in unseren Gesellschaften deutlich zugenommen. Zwischen 1990 und 2010 stieg die Lebenserwartung in Deutschland (und vergleichbar in anderen zentraleuropäischen Ländern) erfreulicherweise von durchschnittlich 75,4 auf 80,2 Jahre an [1]. Betrachtet man allerdings die Anzahl der gesunden Lebensjahre, sieht die Bilanz weniger gut aus. Der Anstieg der gesund verbrachten Lebensjahre betrug nur 3,7 Jahre. Mehr als 1 Jahr der zusätzlichen Lebenserwartung wird somit in Krankheit zugebracht. Als wichtigste Risikofaktoren für die chronischen Erkrankungen wurden ernährungsbedingte Risiken (13,8\%) sowie Bluthochdruck und hoher Body-Mass-Index mit je $10,9 \%$ identifiziert.

Trotz der Erkenntnis, dass durch Beeinflussung der allgemeinen Ernährung und durch Modifikation der Zufuhr von Lebensmittelinhaltsstoffen diese Risikofaktoren vermindert und wesentliche Erfolge in der Behandlung von chronischen Erkrankungen erzielt werden können, ist die heute übliche ärztliche Praxis jedoch weiterhin sehr von der Anwendung spezifischer Medikamente geprägt. Das grosse Potenzial von Lifestyle-Modifikationen, Ernährungstherapien, Gewichtsbeeinflussung und Nährstoffsupplementierung wird von einem grossen Teil der Patienten vernachlässigt, da dieses in der Regel mit erheblichen Änderungen der täglichen Lebens- und Ernährungsgewohnheiten ver- bunden ist. Doch auch von Ärzten wird dieser häufig erklärungsbedürftige und zeitaufwendige Aspekt zu wenig beachtet. Oft wird zusätzlich argumentiert, dass entsprechende Studien (vergleichbar mit Untersuchungen zur Wirksamkeit von Pharmaka) für Lifestyle-Modifikationen nicht vorlägen. Selbstverständlich ist es schwierig und meistens langwierig (und zum Teil auch praktisch nicht möglich), placebokontrollierte Untersuchungen zu Ernährungsänderungen durchzuführen. Darüber hinaus fehlen in der Regel für entsprechende Untersuchungen die notwendigen finanziellen Mittel, da am (möglicherweise positiven) Ende einer Studie kein vermarktbares Produkt, sondern «nur» eine Empfehlung steht. In den vergangenen Jahren hat sich jedoch das Wissen über die Zusammenhänge zwischen Ernährung bzw. einzelnen Nährstoffen und Stoffwechselerkrankungen wesentlich erweitert. Wichtige Aspekte hierzu werden im Rahmen des Themenschwerpunktes «Endokrinologie/Diabetologie/Ernährung» in dieser Ausgabe der SCHWEIZERISCHEN ZeITSCHRIFT FÜR GANZHEITSMEDIZIN anhand der folgenden Artikel dargestellt:

Professor Klaus Kisters, Chefarzt der Klinik für Innere Medizin des St. Anna-Hospitals in Herne und Leiter des dortigen Bluthochdruckzentrums, und Mitarbeiter [2] stellen die Möglichkeiten der Beeinflussung der Hypertonie durch Kochsalzrestriktion sowie erhöhte Zufuhr von Magnesium und Vitamin D dar, wobei die Wirksamkeit einer Vitamin-D-Supplementierung zur Blutdrucksenkung noch umstritten ist. Als Mitglied im Vorstand der Hochdruckliga befasst Prof. Kisters sich seit Langem mit der Bedeutung insbesondere einer ausreichenden Magnesiumzufuhr bei Hypertonie. Viele Untersuchungen belegen inzwischen die grosse Bedeutung einer hohen Magnesiumzufuhr auch bei anderen nichtübertragbaren Krankheiten. Die Häufigkeit von Diabetes mellitus Typ 2, Kopfschmerz (insbesondere Migräne) und Schwangerschaftsproblemen bis hin zu plötzlichem Herztod ist eng mit einem niedrigen Magnesiumstatus verbunden [3]. Neueste Untersuchungen zeigen, dass in unseren westlichen Gesellschaften ca. ein Drittel der Bevölkerung von Magnesiummangel betroffen ist [4].

Einer der am weitesten verbreiteten Risikofaktoren für chronische Krankheiten ist Übergewicht. Leider ist die Gewichtsabnahme und das Halten des geringeren Körpergewichtes jedoch für viele Patienten eine der am schwierigsten umzusetzenden Massnahmen. Kontinuierlich verminderte Kalorienzufuhr führt zu Einspareffekten beim Grundumsatz, wodurch das Abnehmen erschwert wird. Einen möglichen Ausweg aus diesem Dilemma bietet die

\section{KARGER}

(c) 2017 S. Karger GmbH, Freiburg

Fax +497614520714
Prof. Dr. rer. nat. Jürgen Vormann

IPEV - Institut für Prävention und Ernährung

Adalperostrasse 37, 85737 Ismaning, Deutschland

vormann@ipev.de 
Methode des intermittierenden Fastens, insbesondere wenn es mit erhöhter körperlicher Aktivität verbunden ist. Professor Kuno Hottenrott [5], Sportwissenschaftler von der Martin-Luther-Universität Halle/Wittenberg und Präsident der Deutschen Vereinigung für Sportwissenschaft, hat wichtige und neue Untersuchungen zum Erfolg dieser Lifestyle-Massnahmen geleitet und herausgefunden, auf welche zusätzlichen Aspekte geachtet werden muss. Viele Misserfolge von Schlankheitsdiäten sind auf das Nichtbeachten von Änderungen im Säure-Basen-Status durch die verminderte Nahrungszufuhr und die Umstellung des Stoffwechsels hin zum Fettabbau mit nachfolgender Produktion saurer Metabolite zurückzuführen. Diese automatisch entstehende Änderung im Säure-Basen-Haushalt lässt sich jedoch einfach kompensieren, wodurch der Diäterfolg erheblich verbessert wird.

Die am weitesten verbreitete Stoffwechselerkrankung ist natürlich der Diabetes mellitus Typ 2. In Deutschland sind ca. $10 \%$ der Bevölkerung davon betroffen. In der Spätphase der Erkrankung ist die Insulinsubstitution die Ultima Ratio. Allerdings zeigen immer mehr Untersuchungen, dass durch deutliche Gewichtsreduktion nicht nur das Fortschreiten der Erkrankung zum insulinpflichtigen Diabetiker hin verzögert werden kann, sondern möglicherweise sogar eine Heilung erreichbar ist. Professor Stephan Martin [6], Chefarzt für Diabetologie und Direktor des Westdeutschen Diabetes- und Gesundheitszentrums (WDGZ) in Düsseldorf sowie einer der führenden Exper- ten für die Prävention von Herz-Kreislauf-Erkrankungen und die Behandlung von Diabetes mellitus, zeigt Wege dazu auf. Gewichtsreduktion und erhöhte körperliche Bewegung sind natürlich auch hier die Stützpfeiler einer erfolgreichen Lifestyle-Modifikation.

Das brachliegende Potenzial dieser Therapien muss genutzt werden, und der sowohl für den Arzt als auch für den Patienten relativ einfach aussehende Weg der medikamentösen Therapie sollte eine Lifestyle-Modifikation nicht ersetzen.

Stoffwechselerkrankungen nehmen weltweit zu und führen auch zu einer erheblichen finanziellen Belastung der Gesundheitssysteme. Allein durch Anwenden der im Folgenden beschriebenen komplementärmedizinischen Behandlungsmöglichkeiten liessen sich weitverbreitete Gesundheitsprobleme reduzieren und erhebliche Kosten vermeiden.

\section{Literatur}

1 Plass D, Vos T, Hornberg C, et al: Entwicklung der Krankheitslast in Deutschland. Dtsch Arztebl Int 2014;111:629-638.

2 Werner T, Gröber U, Kisters K: Bedeutung der Elektrolyte und Vitamine bei Hypertonie. Schweiz Z Ganzheitsmed 2017;29:DOI: 10.1159/000480131.

3 Vormann J: Magnesium: Nutrition and homoeostasis. AIMS Public Health 2016;3:329-340.

4 Hruby A, McKeown NM: Magnesium deficiency. What is our status? Nutrition Today 2016;51:121-128.

5 Hottenrott K: Intermittierendes Fasten und Sport. Schweiz Z Ganzheitsmed 2017;29:DOI: 10.1159/000480114.

6 Martin S: Lebensstilintervention und Diabetes mellitus Typ 2. Schweiz Z Ganzheitsmed 2017;29:DOI: 10.1159/000480103. 